\title{
Serorreactividad al antígeno de superficie del virus de la Hepatitis B en mujeres que acudieron al Laboratorio Central de Salud Pública. Asunción, Paraguay
}

Gladys Beatriz Olmedo ${ }^{\mathrm{I}}$, María Elena Zorrilla ${ }^{\mathrm{I}}$, María Liz Bobadilla ${ }^{\mathrm{I}}$, Verónica Villagra ${ }^{\mathrm{I}}$, Domingo Santiago Avalos ${ }^{\text {II }}$, Claudia Huber ${ }^{\mathrm{I}}$, Natalia Zarza ${ }^{\mathrm{I}}$

I. Laboratorio Central de Salud Pública. Departamento de Inmunología

II. Departamento de Investigación, dirección de atención primaria de la salud-MSPBS

Cómo referenciar este artículo/ How to reference this article:

\begin{abstract}
Olmedo GB, Zorrilla M, Bobadilla ML, Villagra V, Avalos DS, Huber $\mathbf{C}$, et al. Serorreactividad al antígeno de superficie del virus de la Hepatitis B en mujeres que acudieron al Laboratorio Central de Salud Pública. Asunción, Paraguay. Mem. Inst. Investig. Cienc. Salud. $2015 ; 13(3): 96-102$
\end{abstract}

\section{RE S U M E N}

La hepatitis $B$ es un grave problema de salud pública a nivel mundial, aproximadamente cerca de 2 billones de personas tienen evidencia serológica de infección por el virus de la hepatitis B. El objetivo de este trabajo fue describir la frecuencia de hepatitis B e identificar los factores de riesgo asociados en mujeres en edad fértil que acudieron al Laboratorio Central de Salud Pública entre diciembre de 2013 y junio de 2014. Fue un estudio observacional analítico de corte transverso que, previo consentimiento informado, analizó suero de mujeres entre 15 y 44 años con una edad promedio de $26,6( \pm 6,8)$ años. Mediante la detección del antígeno de superficie de la hepatitis B por ELISA se identificaron seis casos positivos $(0,4 \%)$, indicando una endemicidad baja; cifra que ha variado según perfil socio demográfico: según edad, las de 20 y más años presentaron una frecuencia mayor en comparación a las demás $(p>0,05)$. No se observaron diferencias significativas al evaluar la seropositividad según el estado civil, el nivel de escolaridad, la condición de gravidez, los antecedentes de transfusiones, sin embargo, la seropositividad era mayor en las portadoras de tatuajes/piercing que entre las no portadoras, lo que representaba un riesgo 6,2 veces mayor (OR:6,2 IC95\%:1,3-31,3). En conclusión, la frecuencia del HBsAg en nuestra población es baja, y el factor de riesgo asociado a su detección fue la presencia de tatuajes y/o piercing.

Palabras clave: Hepatitis B, Antígeno de superficie de la hepatitis B, factores de riesgo, marcador serológico para VHB.

\section{Seroreactivity against the hepatitis B virus surface antigen in women who attended the Central Public Health Laboratory. Asunción, Paraguay}

\section{A B S T R A C T}

Hepatitis $B$ is a serious public health problem worldwide; approximately about 2 billion people have serologic evidence of infection with hepatitis B virus. The aim of this analytic cross-sectional study was to describe the frequency of hepatitis $B$ and identify risk factors in women of childbearing age who attended the Central Public Health Laboratory in the period 2013 to 2014. Prior informed consent, antigen detection of hepatitis B surface was performed by ELISA in women between 15 and 44 years with a mean age of $26.6( \pm 6.8)$ years. The identification of six serologic positive cases $(0.4 \%)$ indicates low endemicity. This figure varied according to socio-demographic profile: according to age, those who were 20 years old or older had an increased frequency compared to the others ( $p>0.05)$. 
No significant differences were observed in seropositivity by marital status, level of education, pregnancy, history of transfusion, while seropositivity was higher among carriers of tattoos/piercing than among non-carriers, which represented a 6.2 times higher risk (OR 6.2 95\% CI 1.3 to 31.3). In conclusion, the frequency of HBsAg in our population was low. The risk factor associated with its detection was the presence of tattoos and / or piercings.

Keywords: Hepatitis B, Hepatitis B surface antigen, risk factors, serologic marker for HBV.

\section{INTRODUCCIÓN}

La hepatitis B es un grave problema de salud pública, aproximadamente cerca de 2 billones de personas tienen evidencia serológica de infección por el virus de la hepatitis $B$ (VHB), mientras que cerca de 350 millones de personas tienen infección crónica y alrededor de un millón de estas personas muere cada año por enfermedades hepáticas, incluyendo cirrosis y cáncer hepático (1-4).

La prevalencia de la infección varía en distintas partes del mundo, la OMS definió las áreas de riesgo en América Latina como: endemia baja cuando la seroprevalencia portadora es de 0,5 al 2\% (Centroamérica, Argentina, Paraguay), intermedia cuando es 2 - 7\% (Colombia, Guatemala, Honduras y Cuba), y alta cuando es mayor a 8\% (En la cuenca amazónica de Brasil, Perú, Venezuela, Haití, República Dominicana) (5-8). En los países en vías de desarrollo, la situación se ve agravada por las condiciones sociosanitarias así como también por la deficiencia en los programas de prevención, siendo importante la vacunación en niños menores de un año y en poblaciones de alto riesgo (7-9).

Nuestro país se ha ubicado entre los países de baja endemicidad, basándose en los datos de bancos de sangre con una prevalencia del 0,4\% (5), la cual se encuentra sesgada por varios factores condicionantes como la realización de un pre-tamizaje a través de una serie de preguntas que pueden excluir al infectado y como consecuencia influir en una disminución de la frecuencia de positivos. Sin embargo, es importante resaltar que existen estudios en mujeres en edad reproductiva internadas en el Hospital Psiquiátrico del Ministerio de Salud Pública en Asunción donde no se encontró evidencia serológica de la infección (11). Un estudio realizado en comunidades indígenas Ayoreo del Alto Paraguay, ubicados en zona limítrofe con la ciudad brasileña de Puerto Murtinho arrojó $17,4 \%$ de positividad (12), y otro estudio de una población seleccionada de un servicio universitario de Asunción resultó con 4,5\% de HBsAg positivo (13).

EI VHB es un virus ADN perteneciente a la familia Hepadnaviridae (14). Las vías de transmisión del virus son: relaciones sexuales sin protección, transmisión perinatal o vertical, uso compartido de jeringas y/o agujas contaminadas, percutánea o parenteral a través de lesiones de la piel, corte por objeto punzocortante contaminado, transfusiones de sangre, hemodiálisis, tatuajes, cuando secreciones de personas infectadas entran en contacto con mucosas, procedimientos médicos a través de instrumentos contaminados, y por compartir en forma diaria utensilios (cepillo de dientes, máquinas de afeitar, corta uñas, etc.) de personas con antigenemia positiva (15-17).

El diagnóstico de la hepatitis $B$ se realiza con base en los hallazgos clínicos, epidemiológicos y los estudios de laboratorio. Estos últimos se basan en la cuantificación, a partir de suero o plasma, de diferentes marcadores serológicos que se correlacionan con los diferentes estadios de la enfermedad, y se centra primeramente en la detección del HBsAg, que es un indicador temprano de la presencia de la infección aguda, y al mantenerse positivo por más de 6 meses de portación crónica. Aparece 1-3 meses después de la exposición (18-20). Como consecuencia de que no se realiza rutinariamente el examen serológico para detectar el virus en la población el problema se incrementa, debido a que la mayoría de las personas infectadas son asintomáticas siendo capaces de transmitir la infección (21).

Este estudio tuvo por objetivo determinar la frecuencia de seropositividad al HBsAg del VHB en mujeres que acudieron al Laboratorio Central de Salud Pública (LCSP) en el período 2013 al 2014 e identificar las características sociodemográficas asociadas a la infección, de manera a conocer la magnitud de la enfermedad y poder apoyar las estrategias de prevención y control de la misma con la vacuna contra la hepatitis $B$ (pentavalente: DTP, Haemophilus influenzae tipo b y hepatitis B), siendo la vacunación el 
principal pilar para prevenir la transmisión y evitar la infección $(5,6)$. Además, su detección es el primer tamiz para la identificación de embarazadas portadoras del VHB que permitirá al médico buscar en cada una de ellas, con pruebas adicionales del funcionamiento hepático, evidenciar la afección del hígado así como predecir, tanto el grado de infectividad de las pacientes como la probable evolución, ya que el riesgo de transmisión perinatal oscila entre el $70-90 \%$ en los niños de 6 meses; y si no se realiza la inmunoprofilaxis post-exposición, el $90 \%$ de estos niños permanecerá crónicamente infectado. En niños de 1 a 5 años el riesgo disminuye al $30 \%$ y, los adultos desarrollan infección crónica en un 5-10\% (22-25). También se observa infección fulminante en embarazadas y en los recién nacidos de madres infectadas (26-28).

\section{MATERIALES Y MÉTODO}

\section{DISEÑO}

Se realizó un estudio observacional analítico transversal en el que se incluyeron mujeres entre 15 y 44 años sin antecedentes de vacunación contra la hepatitis $B$, que acudieron al LCSP del MSP y BS, de noviembre del 2013 a junio del 2014. Previo consentimiento informado, se tomaron muestras de sangre y se llevó a cabo una encuesta sobre características socio-demográficas y factores de riesgo asociados a la hepatitis $\mathrm{B}$, y se les extrajo $5 \mathrm{ml}$ de sangre periférica para la detección del HBsAg. La detección del HBsAg se realizó en el departamento de Inmunología del LCSP mediante el método de ELISA HBSAg (Incontrol-Acon Laboratories, Inc. USA) siendo un inmunoensayo enzimático cualitativo con una sensibilidad de $0,2 \mathrm{ng} / \mathrm{mL}$ y la especificidad del $99,9 \%$. Las muestras fueron analizadas por duplicado, se utilizaron controles positivos y negativos para cada placa. La interpretación de los resultados: aquellos sueros con absorbancia por debajo del valor de corte se consideraron negativos, los de absorbancia iguales o mayores que el valor de corte se consideraron positivos. El protocolo de este estudio fue revisado y aprobado por el Comité de Ética del LCSP de acuerdo a lo estipulado en la Declaración de Helsinki, y los datos fueron codificados para mantener la confidencialidad de los mismos.

Los datos fueron digitados con el programa Excel Versión 2010 y el procesamiento de los mismos se realizó en el programa EPI INFO Versión 3.5.4.

En el análisis descriptivo para las variables cuantitativas se aplicaron medidas de tendencia central, y medidas de frecuencias absolutas y relativas para las cualitativas.

Para el análisis bivariado se empleó la prueba de Chi cuadrado con un nivel de significancia $<0.05$. La variable edad se agrupó en dos categorías y se tomó como punto de corte la mediana de edad, el Estado Civil en Solteras y Unión Libre/Casadas; Nivel de Escolaridad en Sin estudios/Primaria y Secundaria/Terciaria, las Gestantes, el Uso de Tatuajes y/o Piercing y los Antecedentes de Transfusión Sanguínea en la categoría de $\mathrm{Si} /$ No.

\section{RESULTADOS}

El promedio de edad de las mujeres estudiadas fue 26,6 años ( $\pm 6,8$ años), el $14,3 \%$ correspondió al grupo de menores a 20 años, observándose mayor frecuencia en las de 20 a 29 años con el 53,2 \%; de ellas el 62,7\% refirió tener pareja estable (Casada/Unión Libre). En cuanto al nivel de escolaridad el $0,3 \%$ se hallaba sin estudio, el $16,0 \%$ correspondía al primario, 55,7 al secundario; el 27,5\% procedía de Asunción y el resto de las demás localidades. El 67,8\% estaba embarazada (Tabla.1). 
Tabla 1. Perfil socio-demográfico y el estado de gravidez de las mujeres que acudieron al LCSP. 2013-2014. $n=1580$

\begin{tabular}{lll}
\hline Variables & $\mathbf{n}$ & $\mathbf{\%}$ \\
\hline $\begin{array}{l}\text { Grupo etario (años) } \\
\quad 20\end{array}$ & 226 & 14,3 \\
$20-29$ & 840 & 53,2 \\
30 a 39 & 429 & 27,1 \\
$\quad \geq 4$ & 85 & 5,4 \\
Estado Civil & & \\
$\quad$ Casada/Unión Libre & 991 & 62,7 \\
Soltera & 589 & 37,3 \\
Nivel de Escolaridad (n=1557) & & \\
Sin estudios & 4 & 0,3 \\
Primaria & 250 & 16 \\
Secundaria & 867 & 55,7 \\
Terciaria & 436 & 28 \\
Procedencia & & \\
$\quad$ Asunción & 434 & 27,5 \\
$\quad$ Otros & 1146 & 72,5 \\
Embarazo & & 67,8 \\
Sí & 1071 & 32,2 \\
No & 509 & \\
\hline
\end{tabular}

Se identificaron seis casos seropositivos al HBsAg siendo la frecuencia de la población estudiada del $0,4 \%$; cifra que ha variado según perfil socio demográfico: según edad, las de 20 y más años presentaron una mayor frecuencia en comparación a las demás, no obstante, esta diferencia no fue significativa $(p=0,6)$.

No se observaron diferencias estadísticas significativas al evaluar la seropositividad según el estado civil, nivel de escolaridad, la condición de gravidez, antecedentes de transfusión, sin embargo, en cuanto a la seropositividad se encontró que era mayor en las portadoras de tatuajes y/o piercing que entre las no portadoras, lo que representa un riesgo 6,2 veces mayor (OR:6,2 IC95\%:1,3-31,3) (Tabla 2). 
Tabla 2. Factores de riesgo asociados a la infección por el virus de Hepatitis B de mujeres que acudieron al LCSP. 2013-2014. $n=1580$

\begin{tabular}{|c|c|c|c|c|c|}
\hline Variables & $\mathbf{n}$ & $\begin{array}{l}\text { Frecuencia } \\
\text { HBsAg }\end{array}$ & $\%$ & OR (IC 95\%) & $\begin{array}{l}\text { Valor } \\
\text { p }\end{array}$ \\
\hline \multicolumn{6}{|l|}{ Edad (años) } \\
\hline$<20$ & 226 & 1 & 0,4 & $1,2(0,1-10,0)$ & 0,6 \\
\hline$\geq 20$ & 1354 & 5 & 0,4 & & \\
\hline \multicolumn{6}{|l|}{ Estado Civil } \\
\hline Casada/Unión Libre & 991 & 5 & 0,5 & $2,9 \quad(0,3-25,5)$ & 0,3 \\
\hline Soltera & 589 & 1 & 0,2 & & \\
\hline \multicolumn{6}{|l|}{ Nivel de Escolaridad } \\
\hline Sin estudios/Primaria & 254 & 2 & 0,8 & $2,5 \quad(0,5-14,1)$ & 0,2 \\
\hline Secundaria/Terciaria & 1303 & 4 & 0,3 & & \\
\hline \multicolumn{6}{|l|}{ Gestante } \\
\hline Sí & 1071 & 4 & 0,4 & & \\
\hline No & 509 & 2 & 0,4 & $0,9 \quad(0,2-5,2)$ & 0,6 \\
\hline \multicolumn{6}{|c|}{ Uso de Tatuajes o Piercing } \\
\hline Si & 219 & 3 & 1,4 & $6,2 \quad(1,3-31,3)$ & $<0,05$ \\
\hline $\begin{array}{l}\text { No } \\
\text { Antecedentes } \\
\text { Transfusión Sanguíne }\end{array}$ & 1361 & 3 & 0,2 & & \\
\hline Si & 55 & 0 & 0 & & \\
\hline No & 1519 & 6 & 0,4 & & \\
\hline
\end{tabular}

\section{DISCUSIÓN}

En este estudio encontramos una seropositividad baja al HBsAg de 0,4\%, lo que coincide con otros realizados en bancos de sangre $(0,38 \%)(10,11)$. Sin embargo, cabe destacar que este porcentaje se basa únicamente en prueba inmunoenzimática de rutina, y no en otros marcadores de infección pero esta prueba resulta ser importante ya que es un indicador temprano para detectar infecciones agudas, ayudando a identificar a los potenciales transmisores de la infección.

Existen estudios en mujeres en edad reproductiva internadas en el Hospital Psiquiátrico del Ministerio de Salud Pública en Asunción donde no se encontró evidencia serológica de la infección (11). Un estudio realizado en comunidades indígenas de Ayoreo del Chaco Paraguayo, ubicados en zonas limítrofes con la ciudad brasileña de Puerto Murtinho arrojó $17,4 \%$ de positividad (12), mientras que otro estudio de una población seleccionada de un servicio universitario de Asunción encontró $4,5 \%$ de positividad para HBsAg (13).

En la población general, especialmente en adolescentes, la presencia de tatuajes y perforaciones es un indicador visible de posibles conductas de riesgo, por lo que existen estudios epidemiológicos que definen la asociación entre tatuajes y/o perforaciones con la infección por el VHB y las enfermedades de transmisión sexual (29). En nuestro estudio se encontró que las mujeres que habían referido tener tatuajes y/o piercing presentaban un riesgo 6,2 veces mayor que las que no poseían, siendo que esta práctica es más frecuente y más aceptada en la población y siendo un procedimiento percutáneo de riesgo, surge la necesidad de adoptar medidas sanitarias reguladoras sobre quienes realizan tatuajes/piercing y en los lugares donde se realizan. Además, se debe reforzar las intervenciones basadas en actividades de promoción, prevención y control sanitario mediante la vacunación contra la hepatitis $\mathrm{B}$ a través del Programa Ampliado de Inmunizaciones. Todas estas son medidas fundamentales teniendo en cuenta el impacto que estos programas han tenido en otras áreas similares o de mayor endemicidad (30).

En conclusión, la frecuencia del HBsAg en nuestra población es baja. No se ha demostrado una relación estadísticamente significativa al evaluar la seropositividad según el estado civil, nivel de escolaridad, la condición de gravidez de las mujeres estudiadas, 
antecedentes de transfusión; pero si se relacionó significativamente con la presencia de tatuajes y/o piercing.

\section{REFERENCIAS BIBLIOGRAFICAS}

1. Organización Mundial de la Salud, 9. Ministerio de Salud Pública del Ecuador. Organización Panamericana de la Salud. La OPS/OMS advierte un aumento en la carga de la hepatitis sobre la salud: Medidas necesarias para mejorar la prevención, la vigilancia, el diagnóstico y el tratamiento /Internet/. Bolivia: OMS/OPS; 2011. /citado 28 jul 2011/. Disponible en: http://www.paho.org/bol/index.php?option=c om_content\&view $=$ article\&id $=1360$ : la-opsoms-advierte-un-aumento-carga-hepatitissobre-saludmedidas-necesarias-prevencionvigilancia-diagnostico-tratamiento\&Itemid $=0$

2. Kwon SY, Lee $\mathrm{CH}$. Epidemiology and prevention of hepatitis B virus infection. [Internet]. /citado 25 marzo 2014/ Korean J Hepatol. 2011;17(2):87-95. Disponible en: http://www.ncbi.nlm.nih.gov/pmc/articles/PM C3304633/pdf/kjhep-17-87.pdf

3. World Health Organization. Hepatitis B vaccines. Weekly epidemiological record /Internet/. 2009 /citado 25 marzo 2014/;84(40):405-20. Disponible en: http://www.who.int/wer/2009/wer8440.pdf? ua $=1$

4. da Costa Castilho M, de Oliveira CM, de Lima Gimaque JB, Leão JD, Braga WS. Epidemiology and molecular characterization of hepatitis B virus infection in isolated villages in the Western Brazilian Amazon. The American journal of tropical medicine and hygiene. 2012 Oct 3;87(4):768-74.

5. Arbo A, Acosta A. Universalización de las vacunas: España. Portugal y países iberoamericanos. Madrid: Real Academia Nacional de Medicina; 2012. Disponible en http://www.coib.cat/uploadsBO//Generica/Do cuments/UNIVERSALIZACION_VACUNAS_489 05.PDF

6. Organización Mundial de la Salud, Organización Panamericana de la Salud. Salud en las Américas. Washington, D.C: OMS/OPS; 2012. Publicación Científica y Técnica No 204.

7. Beltrán Galvis O, Rosas M, Garzón M. Hepatitis B: Diagnóstico y manejo. Revista Colombiana de Gastroenterologia. 2005;20(2):12-33.

8. Asociación Peruana para el Estudio del Hígado, Sociedad de Gastroenterología del Perú, Sociedad Peruana de Enfermedades Infecciosas y Tropicales, Grupo Colaborativo en Hepatitis B. Guía de práctica clínica para el diagnóstico y tratamiento de la hepatitis crónica por el virus de la hepatitis B. Rev. gastroenterol. Perú /online/. 2011 /citado 25 marzo 2014/; 31(2): 151-68. Disponible en:

http://www.scielo.org.pe/pdf/rgp/v31n2/a10 v31n2.pdf

Dirección de Control y Mejoramiento de la Salud Pública. Programa Ampliado de Inmunizaciones. Manual de normas técnicoadministrativas, métodos y procedimientos de vacunación y vigilancia epidemiológica del Programa Ampliado de Inmunizaciones (PAI) /Internet/. Quito: Ministerio de Salud Pública del Ecuador; 2005. /citado 25 marzo 2014/. Disponible en: new. paho.org/ecu/index.php?gid=41\&option =com_docman\&task=doc_download

10. López G, Insfrán I. Coinfección VIH con Hepatitis B (Hvb) y Hepatitis C (Hvc) en el Laboratorio de Referencia del Programa Nacional de Control de VIH/SIDA. Revista del Instituto de Medicina Tropical. 2014 Jul $12 ; 7(1): 19-26 . \quad$ Disponible en: http://www.imt.edu.py/admin/uploads/Docu mento/julio_2012.pdf

11. Rovira CI, Picagua EI, Martínez CI, Ferreira LI, Granado EI, Giménez VI et a. Hepatitis B: antígeno de superficie y anticuerpos post vacunales en mujeres del Hospital Psiquiátrico de Asunción, Paraguay Hepatitis B: surface antigen and post-vaccination antibodies in women from the Psychiatric Hospital of Asunción, Paraguay. Memorias del Instituto de Investigaciones en Ciencias de la Salud. 2011;9(2):57-63. Disponible: http://scielo.iics.una.py/pdf/iics/v9n2/v9n2a0 8.pdf

12. Rovira C, Monzón I, Almirón M. Hepatitis virales en diferentes grupos étnicos del Paraguay. Memorias del Instituto de Investigaciones en Ciencias de la Salud. 2005;3(1):5-8.

13. Rovira C, Picaguá E, Ferreira L, Giménez V, Carpinelli MM, Granado E. Prevalencia de marcadores serológicos de hepatitis virales en una población seleccionada: Experiencia de un servicio universitario. Años 2000-2007. Memorias del Instituto de Investigaciones en Ciencias de la Salud. 2009;7(1):20-6. Disponible en: $<$ http://scielo.iics.una.py/scielo.php?script=s ci_arttext\&pid=S181295282009000100004\&lng=es\&nrm=iso $>$. ISSN 1812-9528.

14. Murray PR, Rosenthal KS, Pfaller MA. Microbiología médica. 5ta. ed. Madrid: Elsevier; 2006.

15. Serrano L, Solano E. Determinación de la inmunidad contra el virus de la hepatitis $b$, en el personal médico del Departamento de Pediatría, Hospital Universitario Dr. Luis Razetti. /Tesis/. /Internet/ Barcelona: Universidad de Oriente; 2008. /citado 19 jun 2014/.

Disponible 
http://ri.bib.udo.edu.ve/bitstream/12345678 9/95/1/Tesis-EM008-S81.pdf

16. Organización Panamericana de la Salud. Control de la difteria, tos ferina, tétanos, haemophilus influenzae tipo b y hepatitis $B$. Washington, DC: OPS; 2006. Guía práctica. No. 604.

17. Ryan KJ, Ray CG. Microbiología médica: una introducción a las enfermedades infecciosas. 4ta. ed. México: McGraw-Hill; 2005.

18. Ministerio de salud. Subsecretaria de Salud Pública. Guía clínica de manejo y tratamiento de la infección por virus de la Hepatitis $B$. Santiago: Minsal 2009.

19. Heathcote J, Abbas Z, Alberti A, Benhamou Y, Chen $C$, Elewaut $A$ et al, revisores. Guías prácticas de la Organización Mundial de Gastroenterología: Hepatitis B. astroenterol. latinoam 2010;21(2):148-64 /Internet/. 2010 /citado 19 jun 2015/. Disponible en: http://gastrolat.org/DOI/PDF/10.0716/gastro lat2010n200005.pdf

20. World Health Organization. Hepatitis B surface antigen assay: operational characteristics (Phase I). /Internet/ Geneva: WHO; 2004. /citado 19 jun 2015/. Available from:

http://www.who.int/diagnostics_laboratory/e valuations/en/hep_B_rep2.pdf

21. Alvarado-Esquivel $C$, Sifuentes-Álvarez A, Pérez-Ochoa J, García-Corral N, RodríguezBriones A, González-Castañeda J, AlonsoMuñozc CM, Bracho-Huemoellera A. Serorreactividad al antígeno de superficie del virus de la hepatitis $B$ en diversos grupos de población de la ciudad de Durango, México. Gac Méd Méx. 2008;144(6):481-4.

22. Hou J, Liu Z, Gu F. Epidemiology and prevention of hepatitis $B$ virus infection. International journal of medical sciences. 2005;2(1):50-7. Disponible en: http://www.medsci.org/v02p0050.pdf

23. Barrera A, Cabrera G, Castañeda A, Montaño A, Peralta R, Serna JS. Diagnóstico y Tratamiento de la infección crónica por virus de hepatitis B. En: Guía de Práctica Clínica, Evidencias y recomendaciones. Catálogo Maestro de Guías de Práctica Clínica: IMSS419-10. México: Secretaria de Salud. 2009.
24. Chen DS. Hepatitis B vaccination: The key towards elimination and eradication of hepatitis B. Journal of hepatology. 2009;50(4):805-16.

25. Abate H, Aquino A, Bakir J, Baran L, Bazan V, Bidone $\mathrm{N}$ et al. Ministerio de Salud de la República Argentina. Recomendaciones Nacionales de Vacunación Argentina. 2012. Disponible en: http://www.msal.gob.ar/images/stories/bes/ graficos/0000000451cnt-201306_recomendaciones-vacunacion-argentina2012.pdf

26. Finelli J, Bell BP. Chapter 4: Hepatitis B. Manual for the Surveillance of vaccinepreventable diseases /Internet/. PD Surveillance Manual, 5th Edition, 2011 Hepatitis B: 4-13 /cited 2013 Jun 15/. Available from: http://www.cdc.gov/vaccines/pubs/survmanual/chpt04-hepb.pdf

27. Ministerio de Salud Pública y Bienestar Social. Programa Ampliado de Inmunizaciones. Enfermedades prevenibles por vacunación. Paraguay. Disponible en: http://www.mspbs.gov.py/pai/index.php?star $t=9$

28. Aspinall EJ, Hawkins G, Fraser A, Hutchinson SJ, Goldberg D. Hepatitis B prevention, diagnosis, treatment and care: a review. Occupational medicine. 2011;61(8):531-40.

29. Pérez-Cotapos ML, Cossio ML. Tatuajes y perforaciones en adolescentes. Revista médica de Chile. 2006 Oct;134(10):1322-9. Disponible

en: http://www.scielo.cl/pdf/rmc/v134n10/art18. pdf

30. Cabezas Sánchez C. Hepatitis virales B y delta: epidemiología y prevención en el Perú. Revista Peruana de Medicina Experimental y Salud Publica. 2002;19(3):150-61. Disponible en: http://www.scielo.org.pe/pdf/rins/v19n3/a09 v19n3.pdf 